\title{
Cross-cultural Comparison of Subjective Well-being (SWB) Predictors Among the Elderly: The Difference Between China and the Other Western Countries
}

\author{
YuXuan Zhang
}

\author{
Beijing World Youth Academy \\ yuxuanzhang211@163.com
}

\begin{abstract}
The current study has investigated the similarities and differences in the predictive effects of subjective well-being (SWB) predictors among the elderly in a range of countries, specifically between China and western countries. The results from a total of 12 studies across more than 20 countries have been compared. Among self-related predictors, the predictive effects of gender, educational level, status of employment, and personality vary across cultures, while age, objective health, and income level do not; among others-related predictors, social support and marital status vary across cultures; among environment-related predictors, household varies across cultures. The difference between collectivist and individualist cultures could reasonably explain all differences across cultures to a certain degree. Future investigations should employ a statistical method for comparison and explore the reasons of the cross-cultural differences in-depth.
\end{abstract}

Keywords: subjective well-being, the elderly, collectivism, individualism

\section{INTRODUCTION}

In the 21 st century, most regions and countries in the world have an aging population. One of the main causes of this phenomenon is the increase in life expectancy [1]. From 2000 to 2019, the global life expectancy has increased from 66.3 to 72.6 years [2], and by $2050,16 \%$ of people globally are projected to be over the age of 65, up from only $9 \%$ in 2019 [3]. As more people are living in their late years, ensuring the subjective wellbeing (SWB) of the elderly has become an increasingly important focus of societies.

In most literatures, SWB was assessed by three dimensions: the tendency of positive affect, the tendency of negative affect, and life satisfaction. However, two studies in China employed slightly different definitions: one literature defined SWB as having four dimensions: positive affect, negative affect, positive experience, negative experience [4]; another literature defined SWB as an individual's emotional and cognitive evaluation of his/her own state of life, which included the life satisfaction, self-realization, pleasure, and tranquility, as well as the appearance of positive affect and the disappearance of negative affect [5]. Positive affect and negative affect were two dimensions of SWB common in all literatures. Despite the difference, the definition of SWB generally shows consistency and so does not affect the comparison in the current study. Based on the existing literatures, SWB in this essay is defined as/measured by the tendency of positive affect and negative affect and life satisfaction.

Self-related, environment-related, and others-related factors were all found to play a role SWB. Most of the previous studies investigated the extent to which these factors predict SWB and the interrelationships between those factors, but only within the specific country/region where the research was conducted. As far as we know, some literatures accounted for the results of their research by the cultural influence in that specific country [6], [7], but few studies actually compared the results cross-culturally. To address this research gap, this study has explored the cultural differences in the predictors of SWB of the elderly. Such a study can provide insights into the specific needs of the elderly who live in different cultural contexts, thus allowing intervention programs to enhance their SWB. The cross-cultural comparison in this study has been undertaken in three categories of predictors: selfrelated, others-related, and environment-related. 


\section{THE CULTURAL DIFFERENCES OF SELF-RELATED PREDICTORS OF SWB}

The first aspect of comparison is the comparison of self-related predictors. There was a general finding across cultures that age was not a significant predictor of SWB and did not impact other predictors. A study in China as well as a study involving a range of participants of different nationalities (Angolan, Brazilian, English, and Portuguese) both supported this finding, suggesting no cultural difference between the significance of age in determining SWB [6], [8].

Apart from age, another basic self-related predictor is gender. One study involving countries in Africa, South America and Europe found no correlation between gender and SWB [8]. By contrast, some studies in China found that SWB was generally higher among males than among females [5], [9]. One study attributed this difference to the higher level of education in males, as level of education was a strong predictor of SWB [9].

When it comes to level of education, which is another predictor of SWB, cross-cultural differences can be identified. One study involving countries in Africa, South America and Europe showed no correlation between level of education and SWB [8], while two other studies in China showed exactly the contrary: SWB was positively correlated with the individuals' level of education [5], [9].

Status of employment seems to predict SWB differently in different countries. SWB was significantly higher among employed elders than among retired elders in a study in China [4]. However, one study based on data collected from 18 European countries obtained a different result: in general, unemployed elders were found to have a higher level of life satisfaction [10].

Studies from Europe and South America both showed that personality was a particularly strong predictor of SWB. A study conducted among Spanish elders as well as a study conducted among Colombian elders both investigated a wide range of predictors of SWB and concluded that personality was the strongest one [11], [12]. Both studies used the Five Factor Model to measure personality trait and found that the most salient predictors were neuroticism, extraversion, and conscientiousness. Neuroticism was the strongest predictor of the negative dimension of SWB, while extraversion and conscientiousness were the strongest predictors of the positive dimensions of SWB. This result suggests a high similarity between the way personality predicts SWB in different cultures. However, personality is not a widely investigated predictor in studies in Asian countries. Apart from age, gender, educational level and personality, objective health was a strong predictor of SWB regardless of region or country. The same held true for income level.

\section{THE CULTURAL DIFFERENCES OF OTHERS-RELATED PREDICTORS}

The second part of the study would explore the cultural differences identified in others-related predictors of SWB. Social support was a widely investigated predictor among studies in China and was found to be positively related to SWB in multiple studies [4], [6], [13]. Furthermore, one study in China found that the SWB of the elderly who received pensions was higher than that of the elderly who received money from their children [5]. Although few studies in non-Chinese regions investigated this predictor, a study encompassing participants from a wide range of regions (Angola, Brazil, England, and Portugal) also found that social support was a significant predictor of SWB [8]. Therefore, social support might be a common predictor cross-culturally with a similar effect on the SWB of the elderly.

Based on two studies in China as well as one study involving participants from 18 European countries, the SWB of the elderly living with spouse was higher than that of the widowed elderly [5], [10], [14]. However, the effect of marital status on SWB seems to be more significant in China [5], [10], [14].

\section{THE CULTURAL DIFFERENCES OF ENVIRONMENT-RELATED PREDICTORS}

In a study involving Angolan, Brazilian, English, and Portuguese elders, household was found to be a negative predictor of SWB, but it was not very contributive to SWB [8]. However, in one study in China, household settings affected SWB significantly. The SWB of the elderly attending senior colleges was higher than the elderly living in homes, welfare homes, and nursing homes. The SWB of the elderly living in homes was higher than those in nursing homes, and the SWB of those living in welfare homes was higher than those in nursing homes [5].

\section{DISCUSSION}

The current study has investigated the cultural differences between predicters of the elderly's SWB. Among self-related predictors, the predictive effects of gender, educational level, status of employment, and personality vary across cultures, while age, objective health, and income level do not. Among others-related predictors, social support and marital status have been found to vary across cultures. Among environmentrelated predictors, household has been found to vary across cultures. 


\subsection{Self-related predictors}

Age was generally not a significant predictor of SWB in studies in different countries [6], [8]. However, another study that investigated the cultural differences in the elderly's SWB predictors and involved a wide range of countries found that SWB decreased with age in countries with a transitioning social organization, but increased in the high-income English speaking countries, along with some other noticeable patterns [15].

Studies in China found that males have a higher SWB than females, while studies in Africa, South America and Europe found less differences across gender [5], [8], [9]. This cross-cultural difference could possibly be explained by the different levels of gender inequality in different countries. One study conducted in regions in China with lower economic status explained that males generally have a higher education level due to the tradition in the Chinese culture of giving more attention and priority to males' education [9]. However, gender inequality in terms of access to and quality of education in recent decades have been vastly improved in China, so this cultural difference may not be salient today.

One study in China found that SWB was positively correlated with the individual's level of education [5], [9], while studies conducted elsewhere mostly found no such correlation [8]. It is possible that in China, the elderly who received higher level of education possess a greater self-care awareness and pay more attention to obtaining enjoyments in life; they tend to read more and actively participate in various cultural and sports activities, thus come into contact with a lot of new things and make friends [5]. On the other hand, lower levels of education may lead to increased loneliness, as suggested by one Chinese study [16]. However, for many individualist western cultures, elders do not need to depend on interactions with others to make life enjoyable, as personal autonomy and individual pursuits are the more common cultural practices [17].

SWB was significantly higher among employed elders than among retired elders in a study in China [4], while in a lot of other European countries, unemployed elders were found to have a higher level of SWB [10]. Accounting for the result obtained in China, the researcher gave the interpretation that the elderly who are unemployed will experience a reduction in social contact with other people such as colleagues and a reduction in social activities after retirement. The scope of the their activities would be limited, and so would their ability to obtain new information. Thus, retired elders are prone to alienation and loneliness [4]. However, the researcher of the study in Europe explained that retirement is traditionally viewed as a life option that relieves an individual's burden from work in a positive way, which suggests a cultural-specific attitude towards retirement different from that in China. This difference can be viewed from the perspective of a collectivist country versus an individualist country [17]. Social support and interaction with others contribute to SWB more significantly for citizens in collectivist countries [17], while employment provides those opportunities. This difference will be further elaborated in the comparison of others-related predictors. The study in Europe also found some variations in their results among its own participants: those who worked under pressure reported a lower level of SWB, but those employed in a job that developed news skills, or those who were self-employed did not experience a low SWB, because the job was rewarding intrinsically for these elders [10]. Yet, the reason that the European elderly experienced a high level of SWB at work was not associated with the presence of social activities or lack of loneliness, which suggests a clear cultural difference between individualist and collectivist countries.

Studies from Europe and South America both showed that personality was a particularly strong predictor of SWB [11], [12]. However, personality was not a widely investigated predictor in studies in Asian countries. This phenomenon might have been produced by the difference between collectivist and individualist cultures. In general, collectivist cultures emphasize "consciousness and greater awareness of and responsiveness to the needs of others" [17]. Meanwhile, individualist cultures emphasize "a sense of personal uniqueness" and thus the expression of one's individuality. Therefore, individual personality is generally less accentuated in collectivist cultures than in individualist ones, which may explain why investigators in China, a collectivist country, do not usually regard personality as a factor that produces individual differences in terms of experience in life. Also, collectivistic cultures usually have more individuals who are allocentric - sharing a collectivist personality trait with others in the same culture [17]. Therefore, again, personality might not be a factor producing individual differences.

\subsection{Others-related predictors}

Across cultures, social support was found to be a predictor of SWB [4], [6], [8]. However, one additional finding in China was that the SWB of the elderly with pensions was higher than that of the elderly who received money from their children [5]. This might have been due to the fact that the elderly with pensions are financially self-sufficient - free to control their own expenses without burdening their children or the society; therefore, they experience less stress [5]. This interpretation is also in line with the values of a 
collectivist culture, where individuals should be aware of and responsive to the needs of others [17].

Marital status was found to affect SWB in some studies in China as well as one study involving participants from 18 European countries; however, the effect seems to be more significant in China [5], [10], [14]. It is reasonable to attribute this difference partly to the collectivist values of Chinese culture which fosters emotional dependence more than European countries [18].

\subsection{Environment-related predictors}

Household was not found to be a significant predictor in Angola, Brazil, England, and Portugul while it was in China [5], [8]. In China, the highest SWB occurred among the elderly who attended senior colleges while the lowest occurred among those living in nursing homes. The study in China explained that the Chinese society traditionally approves of family care as it allows parent-child support, which gives a sense of belonging to the elderly [5]. This could explain the higher SWB and lower levels of loneliness of the elderly living in families than those living in welfare or nursing homes. Welfare and nursing homes create barriers between the elderly and the society and may account for the loneliness experienced by the elderly [5]. In terms of why those in senior colleges experience a high SWB, it is likely that the active environment enables rich social connections while uniting people with a common drive to feel intellectually stimulated, which is closely related to the importance of the sense of solidarity among Chinese elders and in line with norms of the collectivist culture [17]. We can see that for the Chinese elderly, the living environment in general influenced their SWB largely. It is reasonable to think that the environment was partially shaped by the people in the environment, and so it was closely connected to the influence of others-related predictors. The Chinese elderly who tend to develop a sense of self in relation to a group may thus be prone to environmental influences as well.

As a whole, a range of SWB predictors have been found to have different impacts on the elderly across culture. As we can see, the difference between collectivist and individualist cultures could reasonably explain all differences at least to some degree. For members of a collectivist culture, others-related factors such as social support and marital status, status of employment, and household play a huge role in fulfilling the elderly's emotional dependence and sense of interconnectedness [17]. In a collectivist culture, members also emphasize group beliefs over individual beliefs [17], which may explain why gender norms persist, producing a difference in SWB among elderly men and women. On the other hand, members of an individualist culture may not deem level of education, marital status or household as factors that can or should significantly affect their personal pursuits and the fulfilment of personal happiness [17]. In addition, status of employment and personality predicted SWB because they directly concern the elderly themselves. However, it is important to recognize that all cultures have at least certain degree of social connectedness, making social support a universal predictor. We should note that although we have grouped predictors into three categories, it appears that they can all become related to the influence of others depending on our perspective. For members of a collectivist culture, all factors could be related to others, while in individualist cultures, factors such as status of employment may only concern oneself.

\subsection{Future studies}

Future studies can incorporate a wider number and range of literatures from more countries in order to observe more consistent trends in the way predictors of SWB differ across cultures. The results from different studies can be compared statistically. For example, the SWB of the elderly in a country can be correlated with the "individualism versus collectivism" cultural dimension of the country which has been quantified by the website Hofstede Insights [18]. This correlation could provide a more reliable insight into the relationship between culture and SWB. Although the current study ensured that the definition of SWB does no differ across studies in different countries, studies used different scales for measuring SWB. Future studies can try to standardize the scale for a more precise comparison, in case a predictor affects SWB to different degrees in different cultures. Lastly, the current research on SWB of the elderly can be triangulated by other methods, especially interview and longitudinal naturalistic observation. These qualitative research methods can enrich understanding on the subjective experiences of the elderly, their opinions and attitudes, and the meanings they attach to life events, thereby helping us better identify the ways culture influences individual experiences. Literature review like the current one should keep up with the new studies conducted on elders' SWB to align with the rapid cultural changes nowadays. For example, it may be more relevant today to look at the ways in which subtle or underlying social standards on the two sexes affect the SWB of male and female elders, rather than looking at the influence of the difference in educational level among the two sexes.

\section{CONCLUSION}

The investigations of SWB predictors among the elderly from a total of 12 studies across more than 20 countries have been compared. Overall, there is a trend that the Chinese elderly are more affected by predictors 
related to others than the western elderly, which is concordant with the cultural norms in China. This finding should provide insights into the directions of interventions that may be effective specifically among the elderly in China: social support programs, senior colleges, pensions, job opportunities for seniors to bond, and equal opportunities of education among males and females should be things that our society strive to make more available in the following decades. Future studies should continue to specify the needs of the Chinese elderly to improve our understanding on the methods our society should use to improve their SWB.

\section{REFERENCES}

[1] P. J. Dobriansky, R. M. Suzman, and R. J. Hodes, "Why Population Aging Matters - A Global Perspective," US Dep. State, pp. 1-32, 2007, [Online].

Available: papers2://publication/uuid/4B8865DB-5866-4285A74D-168F45ED1109.

[2] E. O.-O. Max Roser and H. Ritchie, "Life Expectancy," Our World Data, 2013.

[3] United Nations, World Population Ageing 2019. 2019.

[4] J. Wu, "The relationship between social support, loneliness and subjective well-being of the elderly." pp. 984-986, 2008.

[5] H. Wang, F., Xu, F., Li, "The status quo of the subjective well-being and loneliness of the elderly." pp. 2533-2535, 2011.

[6] L. Tang, D., Zou, J., Shen, J., \& Zhang, "The Influence Factors of Subjective Wellbeing in Older Adults," Chinese Ment. Heal. J., vol. 20, no. 3, pp. 160-162, 2006.

[7] D. Wang, Y. Tong, L. Zhou, and J. Shen, "The influence mechanism of parent-child support on the subjective well-being of the elderly." pp. 78$82,2004$.

[8] S. Von Humboldt, I. Leal, and F. Pimenta, "Sense of coherence, sociodemographic, lifestyle, and health-related factors in older adults' subjective well-being," Int. J. Gerontol., vol. 9, no. 1, pp. 1519, 2015, doi: 10.1016/j.ijge.2014.01.007.

[9] J. P. Zhang, H. S. Huang, M. Ye, and H. Zeng, "Factors influencing the subjective well being
(SWB) in a sample of older adults in an economically depressed area of China," Arch. Gerontol. Geriatr., vol. 46, no. 3, pp. 335-347, 2008, doi: 10.1016/j.archger.2007.05.006.

[10] H. Axelrad, A. Sherman, and I. Luski, "The effect of employment on the subjective well-being of 6080 years old people," Int. J. Soc. Econ., vol. 47, no. 12, pp. 1481-1497, 2020, doi: 10.1108/IJSE-042020-0212.

[11] I. Etxeberria, I. Etxebarria, and E. Urdaneta, "Subjective well-being among the oldest old: The role of personality traits," Pers. Individ. Dif., vol. 146, no. October 2017, pp. 209-216, 2019, doi: 10.1016/j.paid.2018.04.042.

[12] J. C. Meléndez, E. Satorres, M. A. Cujiño, and M. F. Reyes, "Big Five and psychological and subjective well-being in Colombian older adults," Arch. Gerontol. Geriatr., vol. 82, no. October 2018, pp. 88-93, 2019, doi: 10.1016/j.archger.2019.01.016.

[13] W. Guo and L. Liu, "The Influence of Psychological Capital and Social Support on Old People's Subject Well - being," China J. Heal. Psychol., vol. 22, no. 7, pp. 1054-1056, 2014.

[14] Y. Deng, Z. Yang, and X. Chen, "The correlation between social support and family function and the subjective well-being of the elderly," Chinese J. Gerontol., vol. 32, pp. 3501-3503, 2012.

[15] A. Steptoe, A. Deaton, and A. A. Stone, "Subjective wellbeing, health, and ageing," Lancet, vol. 385, no. 9968, pp. 640-648, 2015, doi: 10.1016/S0140-6736(13)61489-0.

[16] E. T. C. Lai, R. Yu, and J. Woo, "The associations of income, education and income inequality and subjective well-being among elderly in Hong Kong-A multilevel analysis," Int. J. Environ. Res. Public Health, vol. 17, no. 4, pp. 1-14, 2020, doi: 10.3390/ijerph17041271.

[17] V. Karandashev, Cultural Models of Emotions. Springer International Publishing, 2020.

[18] Hofstede Insights, “Country Comparison." https://www.hofstede-insights.com/countrycomparison/ (accessed Aug. 06, 2021). 